Osservazioni di alcuni piccoli pianeti

fatte al R. Osservatorio Astronomico di Milano.

(Equatoriale di $0.218 \mathrm{~m}$ di apertura; distanza focale $3.15 \mathrm{~m}$; micrometro ad anelli; ingrandimento 76 ).

\begin{tabular}{|c|c|c|c|c|c|c|c|c|c|c|}
\hline 1910 & T.m.di Mil. & $A \alpha$ & $A \delta$ & Cf. & $\alpha$ app. & $\log p \cdot A$ & $\delta$ app. & $\log p \cdot A$ & Red. ad l. app. & $*$ \\
\hline
\end{tabular}

(13) Egeria.

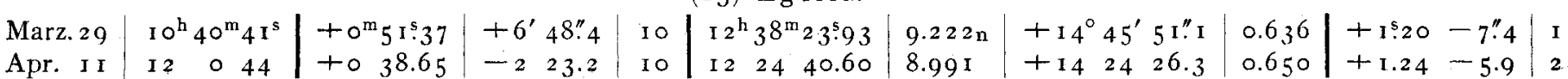
( 198$)$ Ampella.

\begin{tabular}{ll|rrr|rr|rr|r|rrr|r|r|rrr|r|r|r|r} 
Lugl. & I & 10 & 35 & 57 & +0 & 42.43 & +2 & 26.6 & 10 & 18 & 35 & 17.96 & $9.192 n$ & -20 & 6 & 40.6 & 0.910 & +2.27 & -2.7 & 3 \\
& 8 & 9 & 56 & 50 & +0 & 32.82 & -3 & 47.5 & 10 & 18 & 27 & 44.94 & $9.211 n$ & -19 & 34 & 52.4 & 0.909 & +2.32 & -3.0 & 4 \\
& 9 & 10 & 0 & 56 & -0 & 30.88 & +0 & 46.5 & 10 & 18 & 26 & 41.25 & $9.164 n$ & -19 & 30 & 18.3 & 0.907 & +2.33 & -2.9 & 4
\end{tabular} (306) Unitas.

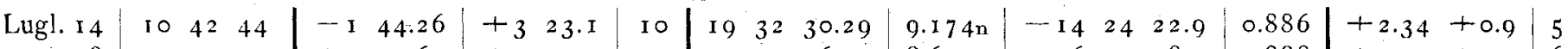
\begin{tabular}{r|rrr|rr|rr|r|rrr|rr|rr|rr|r|r|r}
28 & 10 & 35 & 59 & +3 & 59.65 & +0 & 19.9 & 9 & 19 & 20 & 36.55 & $8.612 \mathrm{n}$ & -16 & 7 & 8.1 & 0.888 & +2.47 & +0.4 & 6 \\
30 & 10 & 43 & 42 & -3 & 7.65 & +2 & 5.3 & 8 & 19 & 19 & 6.89 & $7.953 \mathrm{n}$ & -16 & 22 & 26.3 & 0.888 & +2.49 & +0.7 & 7
\end{tabular}

Posizioni medie delle stelle di riferimento.

\begin{tabular}{|c|c|c|c|}
\hline$*$ & $\alpha \times 910.0$ & $\delta$ Y 910.0 & Autorità \\
\hline I & $\mathrm{I} 2^{\mathrm{h}} 37^{\mathrm{m}} 33^{\mathrm{s}} \cdot 3^{6}$ & $+14^{\circ} 39^{\prime} 10^{\prime \prime} .1$ & $\begin{array}{l}1 / 2\left(A G B e r l A_{4729}+A^{A G}\right. \\
\left.\mathrm{LpzI}_{46} 6_{5}\right)\end{array}$ \\
\hline 2 & I $2 \quad 24 \quad 0.7$ I & +1 42655.4 & Rbg 2788 \\
\hline 3 & $1834 \quad 3.3 .26$ & $-20 \quad 94.5$ & $\begin{array}{c}1 / 3 \text { (II roy } 4650+\mathrm{RC}_{90} \\
4898+\text { DiS } 60 \mathrm{I})\end{array}$ \\
\hline
\end{tabular}

Milano, I 9 I I Magg. 6.

\begin{tabular}{|c|c|c|c|}
\hline$*$ & $\alpha 1910.0$ & $\delta$ I 910.0 & Autorità \\
\hline 4 & ] $8^{\mathrm{h}} 27^{\mathrm{m}} \quad 9^{5} 80$ & $-\mathbf{I} 9^{\circ} 3 \mathrm{I}^{\prime}$ I."9 & $1 / 2\left(\left[\mathrm{Par}_{2}+\mathrm{Par}_{3}\right\} 24 \mathrm{I}_{40}\right)$ \\
\hline 5 & I $934 \quad$ I 2.2 I & $\begin{array}{lll}-14 & 27 & 46.9\end{array}$ & $\mathrm{AG}$ Wa 7380 \\
\hline 6 & $19 \quad 16 \quad 34.43$ & $\begin{array}{lll}-16 & 7 & 28.4\end{array}$ & $v$ Sagittarii F.C. \\
\hline 7 & I $922 \quad 12.05$ & $\begin{array}{lll}-16 & 24 & 32 \cdot 3\end{array}$ & $\mathrm{AG} \mathrm{Wa} 7289$ \\
\hline
\end{tabular}

L. Volta.

Osservazioni del pianeta Interamnia [1910 KU]

eseguite nel R. Osservatorio Astronomico di Capodimonte. Equatoriale di Fraunhofer (o.175m).

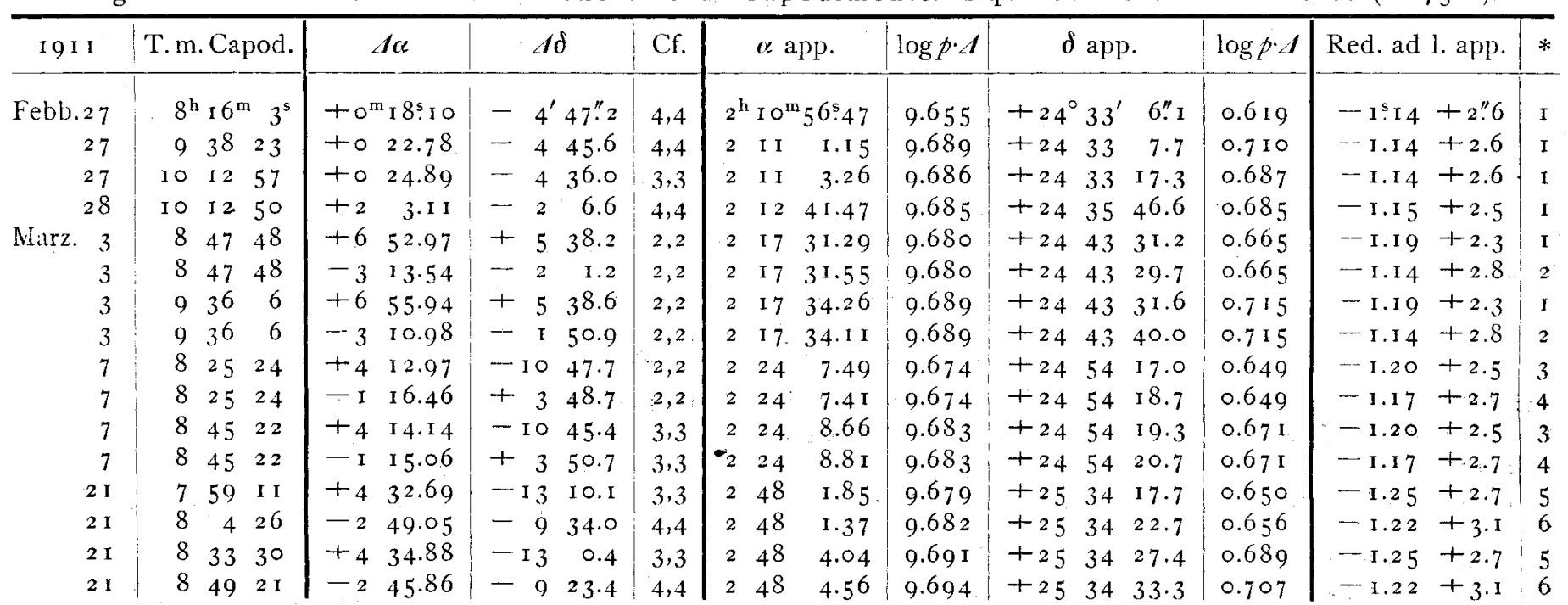

Posizioni medie delle stelle di riferimento.

\begin{tabular}{|c|c|c|c|}
\hline$*$ & $\alpha 1911.0$ & $\delta 1911.0$ & Autorità \\
\hline I & $2^{\mathrm{h}} 10^{\mathrm{m}} 39 \cdot 5 \mathrm{I}$ & $+24^{\circ} 37^{\prime} 50^{\prime \prime} 7$ & AG Berl B $68 \mathrm{I}$ \\
\hline 2 & 22046.23 & $+244528 . \mathrm{I}$ & 734 \\
\hline 3 & 2 I9 55.72 & $+25 \quad 5 \quad 2.2$ & $73 \circ$ \\
\hline
\end{tabular}

\begin{tabular}{|c|c|c|c|}
\hline$*$ & $\alpha 1911.0$ & $\delta$ I 911.0 & Autorità \\
\hline 4 & $2^{\mathrm{h}} 25^{\mathrm{m}} 25^{\mathrm{s}} \cdot 04$ & $+24^{\circ} 50^{\prime} 27^{\prime \prime} 3$ & AG Berl B $75^{2}$ \\
\hline 5 & $\begin{array}{lll}2 & 43 & 30.4 \mathrm{I}\end{array}$ & +254725.1 & AG Cbr F. I 459 \\
\hline 6 & $250 \quad 51.64$ & +254353.6 & ${ }^{1} 516$ \\
\hline
\end{tabular}


Annotazioni.

Febbraio 27, 28. Sereno; immagine del pianeta mediocre. - Marzo 3. Sereno; buona l'immagine del pianeta. Marzo 7. Forte chiarore lunare; pianeta molto debole; osservazioni con appulsi difficilissimi; sereno. - Marzo 2 I. Cielo perfettamente sereno al principio delle osservazioni, con l'immagine del pianeta molto debole; nella seconda metà apparvero densi vapori per cui il pianeta divenna impercettibile e di penosissima osservazione; appulsi molto difficili.

Nel calcolo delle posizioni medie delle stelle di riferimento I) e 4) si è tenuto conto dei moti proprii riportati nel catalogo citato.
I risultati di tutte le osservazioni sono stati corretti dell'errore di orientamento del micrometro, a tal uopo scegliendo opportunamente ogni sera due stelle di paragone; e ciò anche nei giorni 27 e 28 Febbraio nei quali comparisce soltanto la stella AG Berl B 68I, mentre fu anche osservata la 637 dello stesso catalogo per la determinazione dell'orientamento.

Il micrometro che ho sempre adoperato è stato quello lamellare a croce, usufruendo però soltanto degli appulsi corrispondenti all'ingresso dell'immagine del pianeta attraverso le lamine del micrometro; gli altri relativi all'uscita sono stati trascurati poichè, essendo l'immagine del pianeta in generale molto debole, si verificada un sensibile ritardo nella percezione del suo egresso dalla lamina.

Napoli, R. Osservatorio Astronomico di Capodimonte, I 9 I I Maggio 7.

Eugenio Guerrieri.

\section{Wolfscher Komet 1911 a.}

Observations faites à Nice (Equatorial de $0.76 \mathrm{~m}$ d'ouverture).

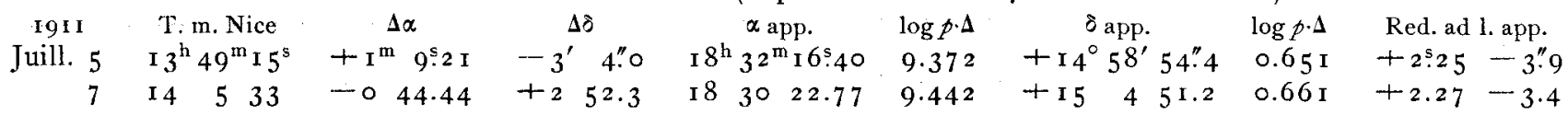

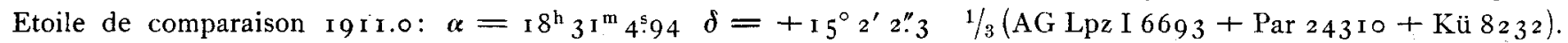

Juill. 5. La comète apparaît comme une petite étoile de grandeur inférieure à la $\mathbf{I}_{4}{ }^{\mathrm{e}}$. Par instants, on aperçoit une petite nébulosité de $\mathrm{I}^{\prime \prime}$ environ d'étendue. Observation difficile. - Juill. 7. La comète conserve le même aspect stellaire que le 5. Elle paraît avoir légèrement augmenté d'éclat.

Nice, I 9 i i Juillet 8.

S. Favelle.

Aus den Sternen AG Lpz II $6849,6855,6867$ und 6869 finde ich den Ort des Kometen für I9 I I Juni I9 I $2^{\mathrm{h}} 4^{\mathrm{m}} \cdot 9$ Kgst. : $\alpha$ I9I1.0 $=18^{\mathrm{h}} 46^{\mathrm{m}} 22^{5} .97 \delta_{1911.0}=+13^{\circ} 29^{\prime}{ }_{16}^{\prime \prime} 7$. Ferner habe ich den Kometen am Reflektor

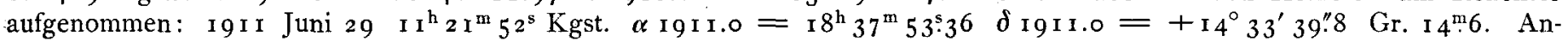
schluß an die Sterne Kü 829 I und AG Lpz I 6753. Der erstere. Stern war nur sehr schwer zu messen, weil zu hell.

M. Wolf.

An meine Elemente $\mathrm{K}_{4}$ (A. N. 186.325) habe ich noch die genäherten Störungen durch Jupiter und Saturn für das Intervall I 9 I I März 28.0 bis Juni 16.0 (Jupiter $\delta M=-16.2$, Saturn $\delta M=-2$.o) angebracht und damit für die

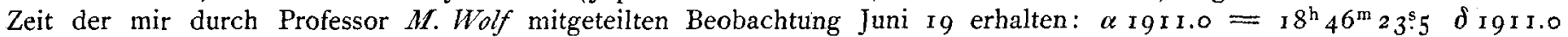
$=+13^{\circ} 29^{\prime} 23^{\prime \prime}$. Die Korrektion der Ephemeride beträgt also nach genäherter Anbringung der Störungen - $0.5-6.3$, Werte, die wohl völlig durch die Anwendung nur 5-stelliger Tafeln erklärt sind. - Ich bitte die Herren Beobachter, die den Wolfschen Kometen beobachten, das Aussehen, die Dimensionen und die Lage des hellsten Teiles des Kometen anzugeben.

M. Kamensky.

\section{Komet 1911 b (Kiess).}

I9I I

Telegramme an die Zentraistelle.

Juli $7 \mathrm{I}^{\mathrm{h}} 39^{\mathrm{m}} \cdot 7$ Mt. Hamilton $\alpha$ app. $=4^{\mathrm{h}} 5 \mathrm{0}^{\mathrm{m}} 5^{\mathrm{s}} \cdot 6 \quad \delta$ app. $=+35^{\circ} 7^{\prime} 4^{\prime \prime} \quad$ Kiess. Corrected pos. from A. N. $45^{\mathrm{I}} \mathrm{I}$. Aitken. 8 I $524.0 \quad 4 \quad 4821.3$

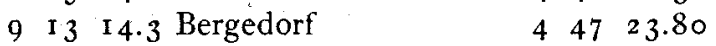
$+345^{8} 43$ Kiess and Aitken.

$+345345 \cdot 3$ Blau, 6 ${ }^{\mathrm{m}} 6$, Schweif schwer zu erkennen, Kopf i' Durchm. ohne sternartigen Kern. H. Thiele.

9 I 426.6 Roma C. R.

$\begin{array}{lll}4 \quad 47 & 15 \cdot 33\end{array}$

$+345243$

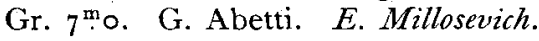

9 I 4 22.5 Simeîs. Photographische Schweiflänge $0^{\circ} 5$ im Pos.-W: $285^{\circ}$. Gr. $4^{\mathrm{m}} \circ$. Grün. Beljawsky. Belopolsky.

I0 I 349.8 Bamberg $\alpha$ app. $=4^{\mathrm{h}} 45^{\mathrm{m}} 35^{\mathrm{s}} .80$ dapp. $=+34^{\circ} 43^{\prime} 55^{\prime \prime}$ Gr. $8^{\mathrm{m}} .5$. Rund, o'6 5 Durchm., Schweifspur. Harturg. I0 I 4 2 I.5 Uccle $4 \quad 45 \quad 3$ I.53 +34443 Gr. 6!5. Biesbroeck. Lecointe.

Beobachtungen.

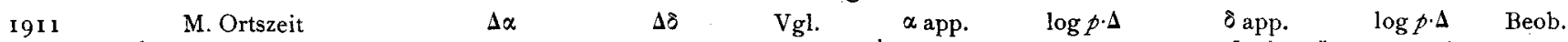

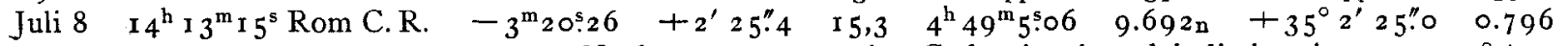
Nucleo $7^{\mathrm{m}} \cdot 5$; testa $\mathrm{I} \cdot 3$; Coda piccola e debolissima in $p=290^{\circ} \pm$. Millosevich. 8 I4 4225 Arcetri $-322.8 \mathrm{I}+\mathrm{I} 58 . \mathrm{I} \quad 8,4 \quad 4492.5 \mathrm{I} 9.698 \mathrm{n}+35$ I 57.7 0.775 A. Abetti. Vergl.-Stern I 9 I r.0: $4^{\mathrm{h}} 5^{2^{\mathrm{m}} 2} 5^{\mathrm{s}} \cdot 5^{2}+34^{\circ} 59^{\prime} 55^{\prime \prime} \cdot 2$ Red. ad l. app. - $0^{\mathrm{s}} 20+4.4$ AG Lei I 8 I 4 , AG Lu 2434. 